\section{Enfermedades raras en la era de la genómica y la medicina de precisión}

\section{Rare diseases in the age of genomics and precision medicine}

\section{Señor editor:}

Aunque existen diversas definiciones, y los países utilizan distintos criterios para su categorización, en general se considera como enfermedades raras a aquellas que afectan a menos de 1 por cada 2.000 habitantes. Estas incluyen trastornos metabólicos, neoplasias, enfermedades nutricionales y neurodegenerativas, entre otras. Aun considerando la baja prevalencia mencionada, se ha reportado que en algunos países, como Estados Unidos, entre 25 a 30 millones de personas podría estar afectada por alguna de estas patologías. Sin embargo, dada la baja frecuencia de cada enfermedad específica, y a pesar de los constantes esfuerzos de los equipos clínicos enfocados en estas materias, la experiencia acumulada en cada situación específica o síndrome suele ser baja, por lo que, incluso los equipos de mayor experiencia, suelen trabajar con altos niveles de incertidumbre ${ }^{1}$.

Desde el inicio de la "Iniciativa de Medicina de Precisión" lanzada el 2015 por el Presidente Obama, cuyo objetivo era incluir 1.000.000 de participantes para realizar un estudio genético de gran escala, así como la iniciativa Británica "Proyecto 100.000 Genomas" lanzada en el 2012 por el Primer Ministro David Cameron, las expectativas han sido altas en cuanto al impacto de los potenciales resultados. En un reciente artículo publicado en Cold Spring Harbor Laboratory se comenta que muchos pacientes con enfermedades raras no reciben un adecuado diagnóstico molecular. No obstante, tras “100.000 Genomas” más de 9.000 pacientes con enfermedades raras han recibido un preciso diagnóstico e incluso se han descubierto nuevas variantes no codificantes causantes de enfermedad ${ }^{2}$. En otro artículo reciente en The Lancet "Research Focus: beyond the 100.000 genomes" se enfatiza que ciertas enfermedades raras podrían ser abordadas de manera precoz y exitosa con la realización de un estudio genético completo. Uno de los problemas con las enfermedades raras es la larga odisea diagnóstica. No es inusual que un paciente vea múltiples consultores y pase años buscando un diagnóstico final, pasando por tres o más diagnósticos erróneos en el camino. Lo que la genómica puede ofrecer es un diagnóstico más rápido y preciso así como ahorrar en tratamientos incorrectamente indicados ${ }^{3}$.

En Chile, en el año 2015 los cánceres constituyeron la segunda causa de muerte después de las cardiovasculares, siendo el cáncer más frecuente para ambos sexos, el de estómago (tasa 18,7 por 100.000 habitantes) seguido del broncopulmonar (17,2 por $100.000)$ y el de colon (10,3 por 100.000). El cáncer se considera una enfermedad genética esporádica, excepcionalmente hereditaria. Solo 5-10\% de los casos de cáncer tienen un patrón de herencia claramente definido. Sin embargo se conocen alrededor de 100 cuadros clínicos de carácter hereditario, asociados a la predisposición a padecer cáncer (Poliposis Adenomatosa Familiar, Síndrome de Lynch, Cáncer de Mama y de Ovario Hereditarios y Neoplasia Endocrina Múltiple, por mencionar algunos). Cabe destacar que muchos de los mayores avances en el conocimiento de los cánceres frecuentes proceden del estudio de síndromes hereditarios raros que predisponen al desarrollo del cáncer ${ }^{4}$.

El estudio de las enfermedades raras tiene un enfrentamiento particular. Desde el punto de vista de clínico, diagnóstico y sanitario, su baja incidencia convierte al caso o a la muestra en el "todo". En este sentido, muchas de las reglas de la medicina basada en la evidencia no aplican para las enfermedades raras y por tanto tampoco los conocidos niveles de evidencia clínica. En el caso de los Biobancos de Investigación de enfermedades raras una pieza o una toma de sangre representan a veces toda la casuística. Vale la pena recordar que las bases que sentaron el desarrollo y creación de las estatinas, uno de los fármacos más prescritos en el mundo, se iniciaron cuando los galardonados con el Premio Nobel de Medicina 1985, Drs. Godlstein y Brown identificaron y estudiaron los fibroblastos de pacientes y familiares con hipercolesterolemia familiar ${ }^{5}$.

La situación actual de las enfermedades raras en nuestro sistema sanitario plantea importantes desafíos estratégicos para el futuro. En primer lugar, resulta imprescindible acumular conocimiento científico de alto nivel con el objetivo de desarrollar medicina de alta precisión que provea a su vez soluciones a las realidades clínicas específicas de los pacientes y sus familias. Por otro lado, será necesario definir políticas públicas explícitas que combinen análisis especializados de tecnologías sanitarias y economía aplicada a la salud. Finalmente, y considerando las experiencias reportadas en Europa y Norte América, se debe promover la investigación científica y el desarrollo tecnológico como una respuesta de la sociedad frente a la realidad de estas enfermedades.

Juvenal A. Ríos Leal ${ }^{1}$, Tomás P. Labbé Atenas ${ }^{2,3}$ ${ }^{1}$ Departamento de Manejo Integral de Cáncer y Otros Tumores, Ministerio de Salud. Santiago, Chile. ${ }^{2}$ Departamento de Enfermedades No Transmisibles, Ministerio de Salud. Santiago, Chile.

${ }^{3}$ Escuela de Medicina, Facultad de Medicina y Ciencia, Universidad San Sebastian. Santiago, Chile. 


\section{Referencias}

1. Berman JJ. Enfermedades raras y medicamentos huérfanos: claves para comprender y tratar las enfermedades comunes. (2015).

2. Ouwehand $\mathrm{W} \mathrm{H}$. Whole-genome sequencing of rare disease patients in a national healthcare system. bioRxiv 507244 (2019). doi:10.1101/507244

3. Watts G. Research Focus: beyond the 100.000 genomes. Lancet 2019; 393: 15-6.

4. Rahner N, Steinke V. Hereditary Cancer Syndromes. Dtsch. Aerzteblatt Online (2008). doi:10.3238/arztebl.2008.0706
5. Cortés V, Vásquez T, Arteaga A, Nervi F, Rigotti A. Rememorando la contribución de Goldstein y Brown al estudio del metabolismo del colesterol: a un cuarto de siglo del Premio Nobel de Medicina. Rev Med Chile 2012; 140: 1053-9.

Correspondencia a:

Dr Tomás Labbé

Departamento de Enfermedades No Transmisibles, Ministerio de Salud.

Enrique Mac Iver 541. Santiago, Chile.

tomas.labbe@minsal.cl 